\title{
Púrpura de Schönlein-Henoch en pediatría: Diez años de experiencia en un consultorio de moderado riesgo en un hospital general
} Henoch-Schönlein purpura in pediatrics: Ten years of experience at a moderate risk office of a general hospital

\author{
Dra. Silvia Gómezza Dra. Mariela Pérez ${ }^{b}$,Dra. Mariana Pellegrinic, Dra. Elsa Isernc, \\ Dra. Cecilia Quintana ${ }^{d}$ Dra. Patricia Artacho ${ }^{a}$, Dra. Margarita Bertolini ${ }^{a}$, \\ Dra. Bettina Pomerantz $z^{a}$ y Dra. Natalia Gadda
}

\section{RESUMEN}

Introducción. La púrpura de SchönleinHenoch (PSH) es la vasculitis leucocitoclástica de pequeños vasos más común en la infancia. Se caracteriza por púrpura palpable y/o compromiso articular, abdominal o renal.

Objetivo: Describir las características epidemiológicas, clínicas, de laboratorio y evolutivas en pacientes con PSH.

Población y métodos. Estudio observacional, descriptivo, retrospectivo. Se incluyeron pacientes menores de 15 años durante el período $1 / 1 / 2008$ al 31/12/2017 que cumplían con los criterios de Ankara para el diagnóstico de PSH. Resultados. Se incluyeron 339 pacientes conPSH; la distribución fue similar en ambos sexos; edad promedio de presentación: 7,02 años $( \pm 3,02)$. El $78 \%$ presentó forma típica y el $22 \%$, atípica, con compromiso articular previoa la aparición de la púrpura. Manifestaciones articulares: el $82 \%$; compromiso abdominal:el $54 \%$; manifestaciones renales: el $19 \%$. El $68 \%$ de los pacientes que tuvieron compromiso renal lo manifestaron dentro del primer mes desde el debut. El síntoma más frecuente fue la hematuria aislada y el $22 \%$ de los pacientes con compromiso renal presentó formas moderadas-graves de nefritis. El $15 \%$ de los pacientes presentó recurrencia/s.

Conclusión. Las manifestaciones articulares fueron más frecuentes de lo descrito en otras series y el compromiso renal fue menor. Si bien este compromiso suele manifestarse en los primeros meses de evolución, se destaca la necesidad del seguimiento de estos pacientes con controles urinarios y de tensión arterial en la evaluación pediátrica.

Palabras clave: Púrpura de Schonlein-Henoch, niños, estudios retrospectivos.

http:/ / dx.doi.org/10.5546/ aap.2020.31

Texto completo en inglés:

http:/ / dx.doi.org/10.5546/ aap.2020.eng.31

Cómo citar: Gómez S, Pérez M, Pellegrini M, Isern E, et al. Púrpura de Schönlein-Henoch en pediatría: Diez años de experiencia en un consultorio de moderado riesgo en un hospital general. Arch Argent Pediatr 2020;118(1):31-37.

\section{INTRODUCCIÓN}

La púrpura de Schönlein-Henoch (PSH) es la vasculitis leucocitoclástica de pequeños vasos más común en la infancia. ${ }^{1-5} \mathrm{Su}$ incidencia es variable según las áreas geográficas. Oscila entre 10 y $20 / 100000$ por año en los menores de 17 años. La edad promedio de presentación es 6 años; predomina en el sexo masculino (relación 2:1) de acuerdo con la mayoría de la bibliografía y se presenta más comúnmente en otoño e invierno. ${ }^{1-3,4,6}$

Se caracteriza por púrpura palpable (condición que debe estar siempre presente) y compromiso articular, abdominal o renal. ${ }^{1,4}$ Raramente, puede afectar otros órganos, como el cerebro, los pulmones, el corazón, los ojos, los testículos, entre otros, dado que es una enfermedad sistémica. ${ }^{7-10}$ Es autolimitada, benigna; se resuelve de manera espontánea en dos semanas en un tercio de los casos, en 2-4 semanas en otro tercio, y el resto, en más de 4 semanas. ${ }^{2,9,11-14} \mathrm{El}$ pronóstico a largo plazo está relacionado con el compromiso renal.

\section{OBJETIVO}

Describir las características epidemiológicas, clínicas, evolutivas y de laboratorio en pacientes con PSH. Analizar factores de riesgo relacionados con el compromiso renal y la recurrencia.

\section{POBLACIÓN Y MÉTODO}

Diseño: observacional, descriptivo, retrospectivo. 
Lugar: Consultorio de Moderado Riesgo, Pediatría Ambulatoria. Período: del 1/1/ 2008 al 31/12/2017.

\section{Criterios de inclusión:}

Pacientes de 1 a 15 años de edad que cumplieron los criterios del Consenso de Ankara (2008) atendidos desde el debut de la enfermedad.

\section{Criterios de exclusión:}

Diagnóstico de otras enfermedades reumatológicas, inmunológicas, hematológicas o renales previas o que estuvieran en tratamiento con medicaciones que pudieran ocasionar púrpura. Pacientes con menos de 2 semanas de seguimiento desde el debut.

\section{Recolección de datos:}

Se revisaron las historias clínicas y se consideraron los criterios del Consenso de Ankara (2008):

- Púrpura comúnmente palpable o petequias, con predominio en los miembros inferiores (MM. II.), no relacionada con trombocitopenia (siempre presente) y, al menos, uno de los siguientes:

- Dolor abdominal difuso de tipo cólico, de comienzo agudo, manifestado por el interrogatorio o por el examen físico. Podía incluir invaginación y sangrado gastrointestinal.

- Artritis de comienzo agudo, definido como dolor con tumefacción o dolor con limitación del movimiento. Artralgia: dolor sin tumefacción y sin limitación del movimiento.

- Compromiso renal: proteinuria $>0,3 \mathrm{~g} / 24 \mathrm{~h}$, cociente albúmina / creatinina en una muestra aislada de orina de la mañana $>30 \mathrm{mmol} /$ mg. Hematuria: > de 5 glóbulos rojos/campo en el sedimento de orina o $2 \mathrm{o}+$ en tiras reactivas. Presencia de cilindros hemáticos en el sedimento.

- Histopatología: vasculitis leucocitoclástica típica con depósitos predominantemente de inmunoglobulina A (IgA) o glomerulonefritis proliferativa con depósitos predominantemente de $\operatorname{Ig} \mathrm{A} .{ }^{2,15}$

\section{Variables:}

Características epidemiológicas: edad, sexo, estación del año.

Características clínicas: antecedentes previos (infecciones respiratorias, medicamentos, inmunizaciones, 15 días antes).
Forma típica: comienzo con púrpura. Atípica: compromiso articular, abdominal o renal previo al comienzo de la púrpura (48 horas antes).

Compromiso articular: artralgia, artritis; abdominal: dolor, vómitos, enterorragia; renal: hematuria, proteinuria, síndrome nefrítico/ nefrótico con o sin caída de filtrado $(<90 \mathrm{ml} /$ $\min / 1,73 \mathrm{~m}^{2}$, estimado según la fórmula de Schwartz). Nefritis leve: hematuria asintomática, macro- o microscópica y / o proteinuria no nefrótica ( $>5 \mathrm{y}<50 \mathrm{mg} / \mathrm{k} /$ día o índice proteína/creatinina - IPC $-<2 \mathrm{mg} / \mathrm{mg}$ ). Nefritis moderada/grave: caída de filtrado glomerular estimado $<90 \mathrm{ml} /$ $\mathrm{min} / 1,73 \mathrm{~m}^{2} \mathrm{y} / \mathrm{o}$ proteinuria persistente en rango nefrótico (> $50 \mathrm{mg} / \mathrm{k} /$ día o IPC $>2 \mathrm{mg} / \mathrm{mg}$ ) con o sin síndrome nefrítico y / o hipertensión arterial (HTA: tensión arterial > Pc 90 según las tablas de la Organización Mundial de la Salud -OMS-).

Análisis de laboratorio inicial: recuento leucocitario, hematocrito, hemoglobina, recuento de plaquetas, proteína $\mathrm{C}$ reactiva $(\mathrm{PCR})$, dosaje de $\operatorname{IgA}$, complemento, eritrosedimentación.

\section{Características evolutivas:}

Tiempo de seguimiento, internación, medicación/es recibida/s.

Recurrencia: paciente con diagnóstico previo de $\mathrm{PSH}$, asintomático por, al menos, cuatro semanas, que presentó nuevamente lesiones cutáneas y/o manifestaciones sistémicas de vasculitis. ${ }^{16,17}$

FIGURA 1. Selección de los pacientes con púrpura de Schönlein-Henoch

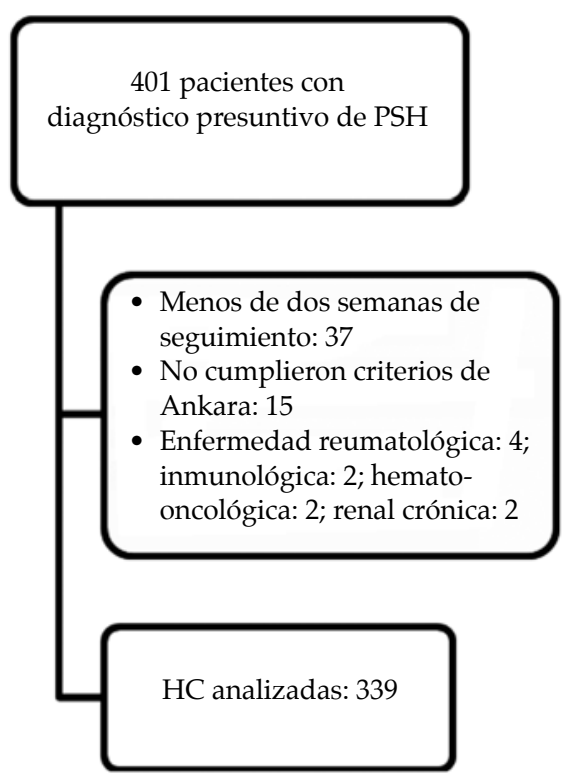

PSH: púrpura de Schönlein-Henoch; HC: historias clínicas. 
Púrpura persistente: pacientes que no estuvieron libres de síntomas desde el diagnóstico inicial hasta un año o más de seguimiento.

\section{Análisis estadístico}

Las variables continuas se presentaron con medidas de tendencia central y de dispersión según la distribución de la muestra. Las variables categóricas se presentaron como frecuencias absolutas y porcentajes. Para la relación entre variables cualitativas, se utilizó la prueba de $\mathrm{chi}^{2}$ / Fisher exact test según la distribución de la tabla de doble entrada. Se consideró un valor de $\mathrm{p}<0,05$ como estadísticamente significativo. Se utilizó, para el análisis, el programa SPSS Statistics V19.

\section{Consideraciones éticas}

El protocolo fue revisado y aprobado por la Coordinación de Docencia e Investigación y el Comité de Bioética Hospitalario.

\section{RESULTADOS}

Se revisaron las historias clínicas de 339 pacientes. Los motivos de exclusión se detallan en la Figura 1. (Promedio: 31 pacientes/ año).

\section{Características epidemiológicas y clínicas:}

La edad promedio de presentación fue 7,02 $\pm 3,02$ años, con distribución similar en ambos sexos (Figura 2). Las características demográficas y antecedentes previos se describen en la Tabla 1. La mediana de tiempo de seguimiento fue de
75 días (rango intercuartil -RIQ-: 40-120). Los casos se presentaron con mayor frecuencia en otoño $(n=107)$, y siguieron en orden decreciente invierno $(\mathrm{n}=86)$, primavera $(\mathrm{n}=81)$ y verano $(\mathrm{n}=65)$.

TABLA 1. Características demográficas y antecedentes previos al inicio de la púrpura de Schönlein-Henoch

\begin{tabular}{lc}
\hline & $\mathbf{N}=339$ \\
\hline Edad al inicio (años) & \\
Media & 7,02 \\
Desvío estándar & 3,026 \\
Sexo, $\mathbf{n}(\%)$ & \\
Masculino & $172(51)$ \\
Femenino & $169(49)$ \\
Tiempo de seguimiento (días) & \\
Mediana & 75 \\
RIQ & $40-120$ \\
Estación del año, n (\%) & \\
Verano & $65(19)$ \\
Otoño & $107(32)$ \\
Invierno & $86(25)$ \\
Primavera & $81(24)$ \\
Antecedentes previos, $\mathbf{n}(\%)\left(\mathbf{N}:\right.$ 329) $\left(^{* *}\right)$ & \\
CVAS & $204(62)$ \\
Estreptococo beta hemolítico & $82(25)$ \\
Mycoplasma & $17(5)$ \\
Medicación & $2(0,6)$ \\
Vacunas & $1(0,3)$ \\
Otros & $4(1)\left({ }^{*}\right)$ \\
Sin antecedentes & $19(6)$ \\
\hline Alimentos, inección gastrontestinal
\end{tabular}

$\left({ }^{*}\right)$ Alimentos, infección gastrointestinal. $\left.{ }^{* *}\right) 10$ sin datos.

CVAS: catarro de vías aéreas superiores; RIQ: rango intercuartil.

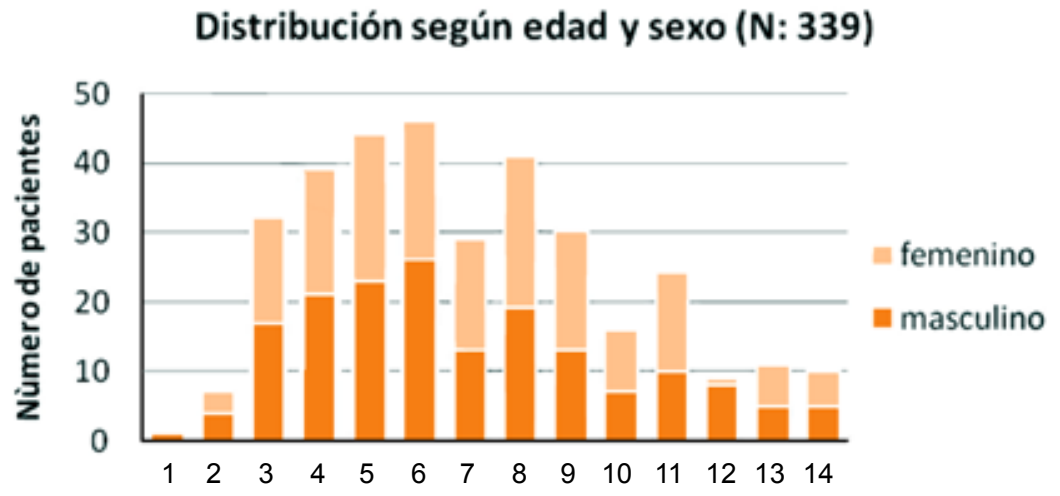

Edad (años) 


\section{Manifestaciones clínicas}

Forma de comienzo: 265 pacientes (el $78 \%$ ) presentaron forma clínica típica y 74 (el $22 \%)$, atípica (Tabla 2). En algunos casos (41; el $12 \%$ ), se constataron síntomas poco usuales, como cefalea $(14 ;$ el $4 \%)$, púrpura ampollar $(7 ;$ el $2 \%)$, compromiso testicular (4; el $10 \%)$, miositis (1; el $0,3 \%)$, conjuntivitis hemorrágica $(1$; el 0,3\%), epistaxis (1; el 3,2 \%), hemoptisis (3; el $1 \%$ ).

Las manifestaciones articulares se observaron en el $82 \%$ de los pacientes, con mayor compromiso de los MM. II., y las articulaciones más afectadas fueron los tobillos y las rodillas (Tabla 3).

El compromiso abdominal se constató en el $54 \%$ de los casos; el dolor fue el síntoma más frecuente. Dos pacientes presentaron invaginación intestinal, confirmada por ecografía y radiología. En estos, el sangrado intestinal fue profuso, mientras que, en el resto, fue escaso e intermitente. El $8 \%$ de los pacientes $(\mathrm{n}=26)$ presentó edema de cuero cabelludo, que requirió tratamiento con corticoides.

La afectación renal se detectó en 65 pacientes (el $19 \%$ ). La hematuria aislada fue la manifestación más frecuente. Se detectó HTA en 14 pacientes; en todos los casos, formó parte de la presentación como síndrome nefrítico. La mayoría de los pacientes (44; el $68 \%$ ) comenzaron con el compromiso renal dentro del primer mes del diagnóstico de PSH y 15 (el $23 \%$ ), dentro de los primeros 2 meses. En el análisis univariado, se encontraron como factores asociados al compromiso renal estadísticamente significativos la edad (> 10 años) $(p=0,004)$, el compromiso abdominal $(p=0,005)$ y la recurrencia $(p=0,000)$.

Los pacientes con nefritis moderada-grave $(\mathrm{n}=14)$, definidos según criterios clínicos,

TABLA 2 Forma de presentación y sintomas al inicio

\begin{tabular}{lc}
\hline Forma clínica: & $\mathbf{N}=\mathbf{3 3 9}$ \\
Típica & $\mathrm{n}(\%)$ \\
Púrpura & $265(78)$ \\
Púrpura, compromiso abdominal & $252(95)$ \\
Púrpura, compromiso articular & $7(3)$ \\
Púrpura, abdominal, articular & $5(2)$ \\
Atípica & $1(0,4)$ \\
Compromiso articular & $74(22)$ \\
Compromiso abdominal & $51(69)$ \\
Articular y abdominal & $21(28)$ \\
Compromiso renal & $1(1)$ \\
\hline
\end{tabular}

fueron tratados conforme el protocolo propuesto por Niaudet modificado: tres pulsos de metilprednisolona $\left(1 \mathrm{~g} / 1,73 \mathrm{~m}^{2}\right)$ endovenosa, seguida de prednisona ( $30 \mathrm{mg} / \mathrm{m}^{2} /$ día $)$ por vía oral durante un mes, luego con $30 \mathrm{mg} / \mathrm{m}^{2}$ días alternos por dos meses $\mathrm{y}$, finalmente, $15 \mathrm{mg} / \mathrm{m}^{2}$ días alternos durante 15 días (Tabla 3). Se indicó ciclofosfamida a razón de $2 \mathrm{mg} / \mathrm{kg} /$ día durante dos meses a los pacientes no respondedores a los corticoides. Presentaron respuesta total, definida como proteinuria normal y filtrado glomerular normal, 8 pacientes; 4 pacientes presentaron respuesta parcial (proteinuria residual, no nefrótica) con filtrado glomerular normal. No respondieron al tratamiento 2 pacientes (persistencia de la proteinuria en rango nefrótico y/o falta de recuperación del filtrado glomerular). De los 2 pacientes no respondedores a los corticoides, uno respondió totalmente a la ciclofosfamida y el restante quedó con proteinuria residual. En 8 pacientes, se realizó una biopsia renal, y el hallazgo histopatológico más frecuente fue la proliferación mesangial focal (III. a Clasificación del International Study of Kidney

TABLA 3. Manifestaciones clínicas

\begin{tabular}{|c|c|c|}
\hline & $\begin{array}{l}\text { Frecuencia } \\
\mathbf{N}=339\end{array}$ & $\begin{array}{c}\text { Porcentaje } \\
(\%)\end{array}$ \\
\hline Púrpura & 339 & 100 \\
\hline Síntomas articulares & 278 & 82 \\
\hline Artralgia & 199 & \\
\hline Artritis & 79 & \\
\hline \multicolumn{3}{|l|}{ Articulaciones afectadas } \\
\hline MM. II. & 257 & \\
\hline MM. SS. & 4 & \\
\hline MM. SS. y MM. II. & 18 & \\
\hline Síntomas abdominales & 182 & 54 \\
\hline Dolor abdominal & 126 & \\
\hline Hemorragia digestiva & 29 & \\
\hline Dolor, vómitos & 27 & \\
\hline Compromiso renal & 65 & 19 \\
\hline Anomalías renales mínimas & 51 & 78 \\
\hline Hematuria aislada & 27 & \\
\hline Proteinuria no nefrótica & 5 & \\
\hline Hematuria y proteinuria no nefrótica & ica 19 & \\
\hline Nefritis moderada/grave & 14 & 22 \\
\hline Sme. nefrótico & 1 & \\
\hline Sme. nefrítico & 1 & \\
\hline Proteinuria nefrótica & 10 & \\
\hline Caída de filtrado & 2 & \\
\hline \multicolumn{3}{|l|}{ Edema } \\
\hline Cuero cabelludo & 26 & \\
\hline Escrotal & 45 & \\
\hline Sacro & 15 & \\
\hline
\end{tabular}

MM. II.: miembros inferiores; MM. SS.: miembros superiores. 
Disease in Children-ISKDC-). ${ }^{18}$ Los pacientes que presentaron proteinuria persistente no nefrótica recibieron inhibidores de la enzima de conversión de angiotensina II $(n=24)$.

Recurrencia: 52 pacientes (el $15 \%$ ) presentaron una o más recurrencias. La mediana del tiempo transcurrido desde el inicio de la enfermedad hasta la aparición de la recurrencia fue de 65 días $(\mathrm{r}=30-540)$. La manifestación clínica más frecuente de la recurrencia fue la púrpura aislada (27; el $52 \%$ ), mientras que el resto presentó púrpura acompañada de otro compromiso. Las variables asociadas a la recurrencia en esta serie fueron el compromiso abdominal $(p=0,006)$ y renal $(p=0,000)$.

Internación: Requirieron internación 63 pacientes (el $19 \%$ ). Los motivos se describen en la Tabla 4.

Recibieron antinflamatorios no esteroides (AINES) 56 pacientes (el 16,5\%), corticoides el $16 \%$. Los AINES fueron utilizados en la mayoría por compromiso articular; los corticoides, por compromiso abdominal grave, compromiso abdominal más articular o por alteraciones renales graves, y los antibióticos, por infección por estreptococo o Mycoplasma.

Purpura persistente: Cuatro pacientes presentaron púrpura persistente durante, al menos, un año de seguimiento, con síntomas intermitentes abdominales y renales; dos de ellos, con hisopado de fauces positivo para estreptococo en varias oportunidades.

\section{Análisis de laboratorio}

Presentaron leucocitosis ( $>15000) 45$ pacientes (el $13 \%)$; plaquetas $>450000 \mathrm{~mm}^{3}: 57$ pacientes (el 16,8\%). PCR: mediana de 0,8 (mg/ dl) (r=0-18); eritrosedimentación: mediana de 15,5 mm/1 h $(\mathrm{r}=2-88)$. En 89 pacientes (el 26\%), se determinó el valor de $\operatorname{IgA}$, que, en el $95 \%$ de los casos, fue elevado para la edad. Los valores de C3 y C4 se obtuvieron en 153 pacientes (el 45\%), en todos los casos con resultado normal. Abandonaron el seguimiento 111 pacientes (el $33 \%$ ).

TABLA 4. Motivos de internación

\begin{tabular}{lcc}
\hline & $\begin{array}{c}\text { Frecuencia } \\
\text { N }=\mathbf{6 3}\end{array}$ & Porcentaje (\%) \\
\hline Compromiso articular & 27 & 43 \\
Dolor abdominal & 14 & 22 \\
Dolor abdominal y enterorragia & 11 & 17,5 \\
Compromiso articular y enterorragia & 9 & 14 \\
Compromiso renal & 2 & 3 \\
\hline
\end{tabular}

\section{DISCUSIÓN}

En esta revisión de 10 años de experiencia de un consultorio de moderado riesgo en un hospital público, se registraron 339 pacientes con diagnóstico de PSH (según los criterios de Ankara). La edad de presentación fue similar a la reportada por diversos autores. Se verificó que, si bien puede presentarse a cualquier edad, es más frecuente en los niños en edad escolar. No se hallaron diferencias según sexo. Esto difiere de otras series en las que ha sido reportada mayor prevalencia en el sexo masculino., ${ }^{3,18}$

La distribución estacional fue similar a lo reportado. ${ }^{2-4,19}$ En dichos estudios, se ha observado que la PSH suele manifestarse más comúnmente durante los meses de otoño e invierno. En más del $80 \%$ de los pacientes, se constató la presencia de patología de vías aéreas superiores. Si bien la causa de la distribución estacional es desconocida, es probable que esté relacionada con la acción de factores desencadenantes, tales como agentes infecciosos.

En todos los casos, se constató la presencia de púrpura (criterio para el diagnóstico). Si bien suele estar presente desde el comienzo, se han descrito formas "atípicas" de la enfermedad en las que la presencia de otros signos y síntomas anteceden a las lesiones en la piel. Estas formas se describen en el $15 \%$ de una serie de 430 niños de entre 2 y 14 años de edad en Turquía ${ }^{2}$ y en un $25 \%$ en otro estudio realizado en Italia $(n=150){ }^{3}$ En nuestra experiencia, se ha encontrado un $22 \%$, que se inició, generalmente, con compromiso articular.

Además del compromiso cutáneo, la PSH se caracteriza por el compromiso de diversos órganos. Las manifestaciones articulares y abdominales han sido reportadas como la segunda y tercera manifestación clínica más frecuentes, pero la prevalencia varía según las publicaciones. El dolor abdominal ha sido evaluado en diversos estudios y varía entre el $35 \%$ y el $50 \%$ de los pacientes, y el compromiso articular, entre el $45 \%$ y el $75 \% .^{2,3,17,19} \mathrm{El}$ compromiso abdominal se registró en la mitad de los niños que fueron asistidos por esta patología durante los 10 años. Se encontró, principalmente, el dolor abdominal como síntoma y, en menor proporción, el sangrado gastrointestinal. La invaginación ha sido reportada como una complicación infrecuente (el $1 \%$ ), lo que concordó con nuestros hallazgos (2 pacientes). Si bien la hemorragia digestiva en estos casos fue profusa, presentaron buena evolución clínica. 
En nuestro trabajo, el compromiso articular fue superior a lo descrito en la literatura. Esta información puede haber sido sobreestimada debido al carácter retrospectivo de la recolección de datos de las historias clínicas. En concordancia con las publicaciones, el compromiso articular se presenta más comúnmente en los MM. II.

El curso clínico de los pacientes con PSH es favorable en la mayoría. La morbilidad en la etapa aguda, así como la necesidad de internación, está relacionada, principalmente, con el compromiso abdominal y /o articular. La morbilidad a largo plazo está relacionada con el grado de compromiso renal. ${ }^{20} \mathrm{Su}$ incidencia varía entre el $10 \%$ y el $60 \%$ de acuerdo con los diversos estudios. 2,3,17,19

En este estudio, se ha detectado la presencia de alteraciones renales en el $19 \%$ de los pacientes, y, en más del $80 \%$ de estos casos, las manifestaciones se iniciaron dentro de los dos meses del debut. Esto último coincide con otros reportes. ${ }^{3,19} \mathrm{La}$ frecuencia que se ha detectado fue, por el contrario, inferior a la reportada por otros autores. ${ }^{2,3,17,19} \mathrm{El}$ tiempo de seguimiento de los pacientes en nuestro estudio tuvo una mediana de 75 días y cerca del $30 \%$ de los pacientes abandonaron el seguimiento, por lo cual las alteraciones renales, que podían presentarse a más largo plazo, pudieron haber sido subevaluadas. En coincidencia con otros estudios, la hematuria fue el hallazgo más frecuente..$^{2,3,19} \mathrm{El} 22 \%$ de los pacientes que presentaron compromiso renal tuvieron nefritis moderada-grave. En la serie descrita, se utilizó el protocolo propuesto por Niaudet modificado, basado en criterios clínicos. Aún existen controversias sobre el tratamiento en estos pacientes.

Diversos factores han sido relacionados con mayor riesgo de compromiso renal: ${ }^{21}$ edad mayor de 10 años, presencia de compromiso abdominal, púrpura persistente y alteraciones en el análisis de laboratorio (leucocitosis, aumento del recuento de plaquetas, antiestreptolisina $\mathrm{O}$ -ASTO-, disminución de C3 y C4). En la serie descrita, se ha encontrado una asociación con la edad (> a 10 años), compromiso abdominal y recurrencias.

Estas últimas han sido mencionadas en diversas publicaciones, con una frecuencia variable: el $7 \%$ en Turquía, el $15 \%$ en España, el $33 \%$ en EE. UU., el $35 \%$ en Italia. ${ }^{3,18,19,22}$ En nuestra serie, se detectaron recurrencias en el $15 \%$ de los casos, con una mediana de 65 días hasta su aparición.
Los análisis de laboratorio no son utilizados para el diagnóstico de PSH. En nuestros datos, se encontró un 13,3\% de leucocitosis, inferior a lo reportado. ${ }^{2,3}$ En la literatura, se mencionan valores aumentados de IgA entre un $20 \%$ y un $50 \% .{ }^{23} \mathrm{Si}$ bien, en los pacientes analizados en este estudio, no se realizó el dosaje de $\operatorname{IgA}$ en forma rutinaria, en aquellos en los que se llevó a cabo, se encontró elevado en el $95 \%$ de los casos.

Una debilidad de nuestro estudio fue la ausencia de datos evolutivos a largo plazo. Por un lado, el tiempo de seguimiento pudo verse afectado por el abandono (aproximadamente, el $30 \%$ de los pacientes) y, por otro, por los cambios en el protocolo de seguimiento que se fueron realizando. En los últimos años, el tiempo se fue prolongando debido a la importancia de continuar pesquisando el compromiso renal luego de la etapa aguda.

El pronóstico de los pacientes con PSH es, generalmente, muy bueno; en ausencia de compromiso renal significativo, la mayoría se recuperan dentro del primer mes de evolución. La mayor parte de los pacientes que presentan compromiso renal lo desarrollan en los primeros dos meses (el $90 \%$ ) y el $97 \%$, dentro de los seis meses. ${ }^{20}$ Sin embargo, el seguimiento debe extenderse durante el primer año de la presentación inicial. Durante los primeros dos meses, es recomendable el control semanal de la tensión arterial y el análisis de orina para luego espaciar estos controles en forma mensual o cada dos meses. Ya que pueden existir manifestaciones tardías, se recomienda que anualmente se realicen controles urinarios y monitoreo de la tensión arterial en la evaluación pediátrica habitual de los niños con el antecedente de PSH.

Se debe monitorear la función renal en los niños con anomalías urinarias, y los que presentan proteinuria persistente, HTA, insuficiencia renal deben ser evaluados por un especialista en Nefrología Infantil. Las mujeres embarazadas con antecedente de PSH deben ser monitorizadas debido al mayor riesgo de presentar HTA. ${ }^{24}$

\section{REFERENCIAS}

1. López Saldaña D. Púrpura de Schönlein-Henoch. Protoc Diagn Ter Pediatr. 2002; 4:81-4.

2. Anil M, Aksu N, Kara OD, Bal A, et al. Henoch-Schönlein purpura in children from western Turkey: a retrospective analysis of 430 cases. Turk J Pediatr. 2009; 51(5):429-36.

3. Trapani S, Micheli A, Grisolia F, Resti M, et al. Henoch Shönlein Purpura in Childhood: Epidemiological and Clinical Analysis of 150 cases over a 5-year period and review of literature. Semin Arthritis Rheum. 2005; 35(3):143-53. 
4. Yildiz C, Ozen S. Childhood Vasculitis. Acta Med. 2013; 2:16-22.

5. Eleftheriou D, Brogan PA. Vasculitis in children. Best Pract Res Clin Rheumatol. 2009; 23(3):309-23.

6. Trnka P. Henoch-Schönlein purpura in children. J Paediatr Child Health. 2013; 49(12):995-1003.

7. Iannetti L, Zito R, Bruschi S, Papetti L, et al. Recent Understanding on Diagnosis and Management of Central Nervous System Vasculitis in Children. Clin Dev Immunol. 2012; 2012:698327.

8. Chen SY, Chang KC, Yu MC, Asueh S, et al. Pulmonary Hemorrhage Associated withHenoch-SchönleinPurpura in Pediatric Patients: Case Report and Review of the Literature. Semin Arthritis Rheum. 2011; 41(2):305-12.

9. Kaur S, Maheshwari A, Aneja S, Seth A, et al. HenochSchönlein purpura with uveitis: an unusual case and review of literature. Rheumatol Int. 2012; 32(12):4057-9.

10. Migita M, Hayakawa J, Shima H, Kobayashi H, et al. A Case of Henoch-Schönlein Purpura with rare complications: necrosis of the small intestine, neurological symptoms, and pericardial tamponade. J Nippon Med Sch. 2005; 72(6):383-6.

11. McCarthy H, Tizard E. Diagnosis and management of Henoch-Scönlein purpura. Eur I Pediatr. 2010;169(6):643-50.

12. Campos SR. Púrpura de Schönlein-Henoch. Protoc Diagn Ter Pediatr. 2014; 1:131-40.

13. Smith G. Management of Henoch-Schönlein purpura. J Pediatr Child Health. 2012; 22(8):327-31.

14. Jauhola O, Ronkainen J, Koskimies O, Ala-Houhala M, et al. Clinical course of extrarenal symptoms in HenochShönlein purpura: a 6-month prospective study. Arch Dis Child. 2010; 95(11):871-6.

15. Rahman R, Cobeñas C. Púrpura de Schönlein-Henoch.
En Comité Nacional de Nefrología Pediátrica. Nefrología Pediátrica. Buenos Aires: FUNDASAP; 2008.Págs.226-30.

16. AlfredoCS, Nunes NA, Len CA, BArbosa CM, etal.HenochSchönlein purpura: recurrence and chronicity. J Pediatr (Rio J). 2007; 83(2):177-80.

17. Praism D, Amir J, Nussinovitch M. Recurrent HenochSchönlein purpura in children. J Clin Rheumatol. 2007; 13(1):25-8.

18. Niaudet $P$, Habib R. Methylprednisolone pulse therapy in the treatment of severe forms ofSchönlein-Henoch purpura nephritis. Pediatr Nephrol. 1998; 12(3):238-43.

19. Saulsbury F. Henoch-Schonlein purpura in children. Report of 100 patients an review of the literature. Medicine (Baltimore). 1999; 78(6):395-409.

20. Narchi H. Risk of long term renal impairment and duration of follow up recommended for Henoch-Schönlein purpura with normal or minimal urinary findings: a systematic review. Arch Dis Child. 2005; 90(9):916-20.

21. Chan H, Tang YL, Lv XH, Zhang GF, et al. Risk Factors associated with Renal Involvement in Childhood HenochSchonlein Purpura: a Meta-Análisis. Plos One. 2016; 11(11): 0167346.

22. Ángeles L, Ballona R. Púrpura de Henöch- Schönlein: epidemiología, clínica y seguimiento por cinco años en pacientes hospitalizados en el Instituto Especializado de Salud del niño, 1995-1999. Folia Dermatol. 2007; 18(3):111-7.

23. Piram M, Mahr A. Epidemiology of immunoglobulin A vasculitis (Henoch-Schönlein): current state of knowledge. Curr Opin Rheumatol. 2013; 25(2):171-8.

24. Ronkainen J, Nuutinen M, Koskimies O. The adult kidney 24 years after childhood Henoch-Schönlein purpura: a retrospective cohort study. Lancet. 2002; 360(9334):666-70.

\section{Artículos seleccionados}

Los siguientes resúmenes y comentarios de trabajos seleccionados se encuentran disponibles en la versión electrónica de este número.

Lancet Child Adolesc Health. 2019;3(6):408-17

Edad gestacional al nacer y mortalidad desde la infancia hasta la edad adulta media: estudio de cohorte nacional (Crump C, et al. Gestational age at birth and mortality from infancy into mid-adulthood: a national cohort study)

Comentario: Dr. Ramón Larcade. Neonatología, Sanatorio de la Trinidad Ramos Mejía.

Dr. Ricardo Ciammella. Unidad de Medicina Fetal, Hospital Universitario Austral. Buenos Aires

Pediatrics. 2019 Sep;144(3). pii: e20190844.

Satisfacción de los pacientes y prescripción de antibióticos por telemedicina en infecciones respiratorias (Foster $C B$, et al. Patient satisfaction and antibiotic prescribing for respiratory infections by telemedicine)

Comentario: Dr. Adrian Bordas Perez. Hospital Universitario Arnau de Vilanova, Lleida, España. Dr. Norberto Giglio. Epidemiologia Hospital de Niños Ricardo Gutiérrez, Ciudad de Buenos Aires, Argentina,

Pediatr Pulmonol. 2019; 54(5):525-530.

Evolución clínica, funcional y radiológica en niños con empiema pleural (Maffey A, et al. Clinical, functional, and radiological outcome in children with pleural empyema)

Comentario: Dr. Conrado J. Llapur. Hospital del Niño Jesús, Tucumán. 